\title{
Quantum phase transition of polaritonic excitations in a multi-excitation coupled array
}

\author{
Lituo Shen ${ }^{1}$, Rongxin Chen ${ }^{1}$, Huaizhi Wu ${ }^{1}$, Zhenbiao Yang ${ }^{1}$, E. K. Irish ${ }^{2}$, and Shibiao Zheng ${ }^{1 *}$ \\ ${ }^{1}$ Fujian Key Laboratory of Quantum Information and Quantum Optics, College of Physics \\ and Information Engineering, Fuzhou University, Fuzhou, Fujian 350116, China \\ ${ }^{2}$ Physics and Astronomy, University of Southampton, Southampton SO17 1BJ, UK
}

\begin{abstract}
We analyze the quantum phase transition-like behavior in the lowest energy state of a two-site coupled atomcavity system, where each cavity contains one atom but the total excitation number is not limited to two. Under the large-detuning condition, we identify an interesting coexisting phase involving characteristics of both photonic superfluid and atomic insulator, which has not been previously revealed. For small hopping, we find that the signature of the photonic superfluid state becomes more pronounced with the increase in total excitation number, and that the boundaries of the various phases shift with respect to the case of two excitations. In the limit of small atom-field interaction, the polaritonic superfluid region becomes broader as the total excitation number increases. We use alternative order parameters to characterize the nonclassical property in the lowestenergy state, and find that the entanglement of photons in the photonic superfluid state has an approximately quadratic-like dependence on the total excitation number within the large-detuning limits. The second-order cross-correlation function is demonstrated to become inversely proportional to the total excitation number in the large detuning limits.
\end{abstract}

PACS numbers: 42.50.Pq, 05.70.Fh, 03.67.Lx

\section{INTRODUCTION}

Strongly correlated phenomena in controllable quantum many-body systems have attracted great attention in optical lattices [1, 2] and Josephson-junction arrays [3]. One of the simplest and most important models describing these lightmatter interactions is the Jaynes-Cummings-Hubbard (JCH) model [4-6], which describes a coupled array of cavities each containing a two-level system. Similar to the Bose-Hubbard (BH) model [7, 8] used for cold atoms in an optical lattice, the JCH model exhibits the photon blockade effect $[9,10]$ and the Mott-insulator-to-superfluid quantum phase transition [11].

Coupled cavity-QED arrays can operate at high temperatures and allow for individual site addressing. These features, together with progress in realizing the strong light-matter coupling regime in both atomic and solid-state systems [12-14], are attracting more and more attention to the quantum phase transitions in coupled-cavity arrays captured by the JCH model. These quantum phase transitions are due to the transfer of excitations from polaritonic states to photonic states, where polaritons are superpositions of photons and excitations of the atoms or atom-like structures, rather than purely bosonic or purely fermionic excitations. Most previous studies related to quantum phase transitions in coupled-cavity arrays have focused on the large site number [15] and large atom number limits [16-19], which are analogous to the purely bosonic B$\mathrm{H}$ model and can be analytically solved within the mean-field approximation.

Recent research shows evidence that novel quantum phase transition-like behavior may appear in finite systems involving a very few interacting sites and a small number of twolevel systems [16, 20-56], where the transition behavior becomes dependent on the number of sites and two-level system-

*Electronic address: sbzheng11@163.com s. Greentree et al. [4] showed that the Mott-insulator to superfluid quantum phase transition could appear in a mesoscopic two-dimensional coupled array. Hartmann et al. [5] used a four-level atom to simulate the effective on-site potential and the Mott-insulator to superfluid phase transition. Angelakis et al. [6] considered the simulation of an $X Y$ spin model based on the Mott regime in a linear array of cavities, each containing a two-level atom and a photon. Makin et al. [15] studied the phase diagrams of the system composed of a few photonic cavities, each containing a two-level atom, but the changes in the nature of the excitations accompanying the phase transition remaining untouched upon. Irish et al. [20] demonstrated phase transitions of polaritonic excitations in a two-site, twoexcitation coupled array. In a very recent ion-trap experiment [57], Toyoda et al. reported the simulation of the quantum transition of polaritonic excitations in a JCH model using two trapped ions and phonons within the two-excitation Hilbert subspace. Previous works on the two-site coupled array model were limited to the two-excitation Hilbert subspace without considering the situation with higher excitation numbers in the photonic states. It is known that the number of photons plays an important role in the coefficient of on-site repulsion for small finite systems [4], but the influence of extra photons on the quantum phase transition in the lowest-energy state of the coupled atom-cavity system is still unclear.

In this work, we investigate quantum phase transition-like behavior in the lowest-energy state (within the $N$-excitation Hilbert subspace) of a two-site coupled atom-cavity system, where each cavity contains one atom but the total excitation number $N$ is not limited to two. (Note that the description$\mathrm{s}$ " insulator" and "superfluid" used in this paper represent the localized and delocalized states in the small finite system we consider. Such an investigation can also be generalized to larger arrays, in which the localization-delocalization transitions studied here approach genuine quantum phase transitions.) More importantly, we find that for the four-excitation case there exists an interesting phase, in which the atoms ex- 
hibit the insulator characteristic while the photons show the superfluid behavior. For the two-excitation case, such a phase does not appear and, as far as we know, has not been discovered. Equilibrium states of a Jaynes-Cummings Hubbard model have been demonstrated in the recent ion-trap setup [57], where the photons are replaced by phonons. We alternatively use the order parameters of entropy, the Mandel Qparameter, and the second-order cross-correlation function to reveal the nonclassical property of the lowest-energy state in the multi-excitation coupled array, and establish their possible correspondence with the phase diagram, and find interesting effects caused by the total excitation number on their nonclassical properties.

\section{RESULTS}

\section{A. System and Hamiltonian.}

We consider the system consisting of two sites, each supporting a field mode and containing a single atom. Photons are able to hop between these two field modes. Under the rotating-wave approximation, our system is governed by the following Hamiltonian $(\hbar=1)$ :

$$
\begin{aligned}
H= & \sum_{j=1,2}\left[w_{c} a_{j}^{\dagger} a_{j}+w_{a}\left|e_{j}\right\rangle\left\langle e_{j}\right|+\lambda\left(a_{j}^{\dagger}\left|g_{j}\right\rangle\left\langle e_{j}\right|\right.\right. \\
& \left.\left.+a_{j}\left|e_{j}\right\rangle\left\langle g_{j}\right|\right)\right]+h\left(a_{1}^{\dagger} a_{2}+a_{1} a_{2}^{\dagger}\right),
\end{aligned}
$$

where $a_{j}^{\dagger}$ and $a_{j}$ are the creation and annihilation operators of the $j$ th field mode with frequency $w_{c} .\left|e_{j}\right\rangle$ and $\left|g_{j}\right\rangle$ represent the excited and ground states of the $j$ th atom with frequency $w_{a} . \lambda$ is the atom-field coupling strength and $h$ is the strength of the hopping between the two cavity fields. Note that the excitation number of the total system is conserved since the excitation number operator $\hat{N}=\sum_{j=1,2}\left(\left|e_{j}\right\rangle\left\langle e_{j}\right|+a_{j}^{\dagger} a_{j}\right)$ commutes with the Hamiltonian $H$. As is well known, when the field is in state for which the dominant frequency is nearly close to a particular atomic transition and we may neglect interaction terms with very high frequency, the rotating-wave approximation is valid. This approximation assumes that the field strength itself is not too large and the state of the field does not vary rapidly on a time scale of a particular atomic transition frequency, i.e., fields of very fast strong pulses are ignored. However, the large detuning and different hopping conditions in our paper are both compared with the atom-field coupling strengthes, and the rotating-wave approximation is still satisfied here under large detuning and different hopping conditions. Therefore, the analysis of our results are valid for the Hamiltonian of Eq. (1).

\section{B. Total excitation number $N=4$.}

\section{Small hopping.}

When there is no photon hopping between the two sites, the eigenstates in each site are given by the polaritonic states [20]

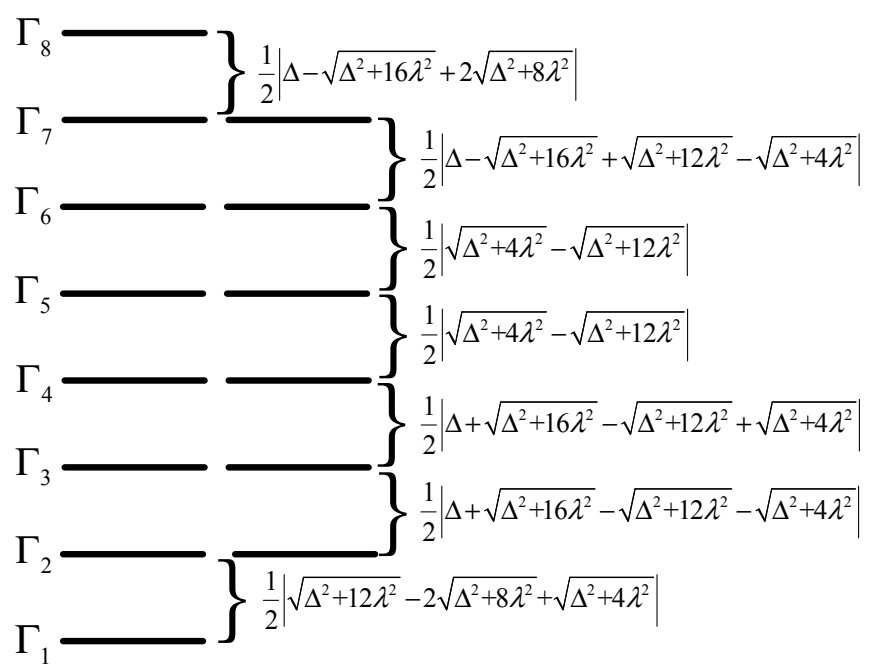

FIG. 1: Energy-level difference between two nearest neighbor subspaces when there is no photon hopping and $N=4$.

$$
\begin{aligned}
\left|0_{j}\right\rangle & =\left|g_{j}\right\rangle\left|0_{j}\right\rangle, \\
\left|n_{j}^{-}\right\rangle & =\sin \left(\frac{\theta_{n}}{2}\right)\left|e_{j}\right\rangle\left|(n-1)_{j}\right\rangle-\cos \left(\frac{\theta_{n}}{2}\right)\left|g_{j}\right\rangle\left|n_{j}\right\rangle, \\
\left|n_{j}^{+}\right\rangle & =\cos \left(\frac{\theta_{n}}{2}\right)\left|e_{j}\right\rangle\left|(n-1)_{j}\right\rangle+\sin \left(\frac{\theta_{n}}{2}\right)\left|g_{j}\right\rangle\left|n_{j}\right\rangle,
\end{aligned}
$$

where $j=1,2$. $\left|n_{j}\right\rangle(n=1,2,3, \ldots)$ represents the Fock state of the $j$ th field mode, and $\tan \left(\theta_{n}\right)=2 \lambda \sqrt{n} / \Delta$, where the detuning $\Delta=w_{a}-w_{c}$. The corresponding energies for these eigenstates are:

$$
\begin{aligned}
E_{j}^{0} & =0, \\
E_{j}^{n^{-}} & =n w_{c}+\frac{\Delta}{2}-\frac{1}{2} \sqrt{\Delta^{2}+4 n \lambda^{2}}, \\
E_{j}^{n^{+}} & =n w_{c}+\frac{\Delta}{2}+\frac{1}{2} \sqrt{\Delta^{2}+4 n \lambda^{2}} .
\end{aligned}
$$

Unlike the previous study in Refs. [20, 57] where the analysis is restricted to the Hilbert subspace with only two excitations, our analysis here focuses on the insulator-superfluid quantum phase transition in the multi-excitation Hilbert space, i.e., the total excitation number $N$ can be larger than two. For simplicity, we consider the total excitation number to be an even number, for which the lowest-energy state is nondegenerate. In the following, we begin the analysis of quantum phase transition in the lowest-energy state of our atom-cavity system for the case $N=4$ and later generalize it to $N>4$.

When $h=0$ and $N=4$, the eigenstates of the system are, arranged in order of increasing energy,

$$
\begin{aligned}
& \Gamma_{1}=\left\{\left|2_{1}^{-}\right\rangle \otimes\left|2_{2}^{-}\right\rangle\right\}, \\
& \Gamma_{2}=\left\{\left|1_{1}^{-}\right\rangle \otimes\left|3_{2}^{-}\right\rangle,\left|3_{1}^{-}\right\rangle \otimes\left|1_{2}^{-}\right\rangle\right\},
\end{aligned}
$$



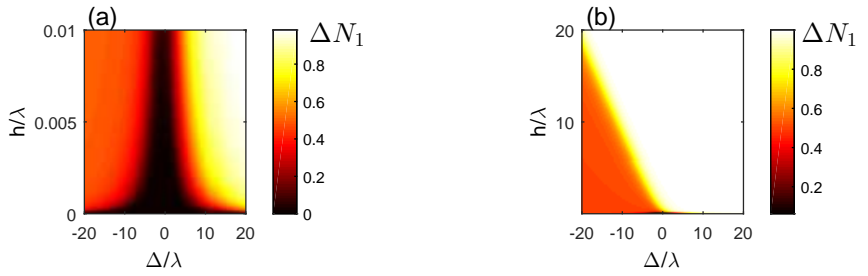

FIG. 2: (Color online) [(a)-(b)] $\Delta N_{1}$ as a function of $\Delta$ and $h$ for the lowest-energy state of the system when $N=4$.

$$
\begin{aligned}
& \Gamma_{3}=\left\{\left|0_{1}\right\rangle \otimes\left|4_{2}^{-}\right\rangle,\left|4_{1}^{-}\right\rangle \otimes\left|0_{2}\right\rangle\right\}, \\
& \Gamma_{4}=\left\{\left|1_{1}^{+}\right\rangle \otimes\left|3_{2}^{-}\right\rangle,\left|3_{1}^{-}\right\rangle \otimes\left|1_{2}^{+}\right\rangle\right\}, \\
& \Gamma_{5}=\left\{\left|2_{1}^{+}\right\rangle \otimes\left|2_{2}^{-}\right\rangle,\left|2_{1}^{-}\right\rangle \otimes\left|2_{2}^{+}\right\rangle\right\}, \\
& \Gamma_{6}=\left\{\left|3_{1}^{+}\right\rangle \otimes\left|1_{2}^{-}\right\rangle,\left|1_{1}^{-}\right\rangle \otimes\left|3_{2}^{+}\right\rangle\right\}, \\
& \Gamma_{7}=\left\{\left|4_{1}^{+}\right\rangle \otimes\left|0_{2}\right\rangle,\left|0_{1}\right\rangle \otimes\left|4_{2}^{+}\right\rangle\right\}, \\
& \Gamma_{8}=\left\{\left|2_{1}^{+}\right\rangle \otimes\left|2_{2}^{+}\right\rangle\right\} .
\end{aligned}
$$

Figure 1 shows the energy differences between consecutive energy levels.

Note that the energetic ordering of the subspaces $\Gamma_{1} \rightarrow \Gamma_{8}$ is independent of the parameters $\lambda$ and $\Delta$. Notably, the gap between the lowest two energy levels is $E_{\Delta_{1,2}}=\frac{1}{2} \mid \sqrt{\Delta^{2}+12 \lambda^{2}}-$ $2 \sqrt{\Delta^{2}+8 \lambda^{2}}+\sqrt{\Delta^{2}+4 \lambda^{2}}$, which approaches zero in the limits of both large positive and large negative detuning. This is contrary to the situation with total excitation number $N=2$, in which the energy difference between the lowest two energy levels goes to infinity in the limit of large negative detuning $[20,57]$.

In order to distinguish the insulator and superfluid states in the lowest-energy state of the system, we use the variance of the total excitation number on the first site $\hat{N}_{1}$ as a measure [6]:

$$
\Delta N_{1}=\left\langle\hat{N}_{1}^{2}\right\rangle-\left\langle\hat{N}_{1}\right\rangle^{2}
$$

where $\hat{N}_{1}=a_{1}^{\dagger} a_{1}+\left|e_{1}\right\rangle\left\langle e_{1}\right|$. In the insulator state $\Delta N_{1}$ is zero, while in the superfluid state $\Delta N_{1}$ is nonzero. A plot of $\Delta N_{1}$ as a function of the detuning $\Delta$ and the photon hopping strength $h$ is given in Fig. 2.

For $\Delta=0$ and $h / \lambda<<1$, the atom-field interaction on one site shifts the frequency of the field, causing a photon blockade effect $[9,10]$ that leads to a large energy gap between the lowest two subspaces $\left(\Gamma_{1}\right.$ and $\left.\Gamma_{2}\right)$ and prevents additional photons from entering the site. For $N=2$ the gap between the lowest two energy levels is given by [20] $(2-\sqrt{2}) \lambda \simeq 0.59 \lambda$. For $N=4$ the corresponding gap is $(2 \sqrt{2}-1-\sqrt{3}) \lambda \simeq 0.096 \lambda$, indicating that the hopping strength $h$ needed to overcome the photon blockade is much smaller as compared with the case with $N=2$. For $N=4$, the lowest-energy state of the system is approximated by $\left|2_{1}^{-}\right\rangle \otimes\left|2_{2}^{-}\right\rangle$, as shown in Fig. 3(a), where $N_{A}$ is defined as the total excitation number of both atoms. This
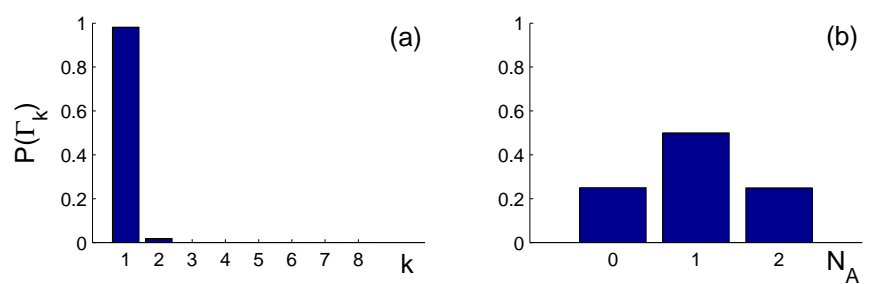

FIG. 3: (Color online) (a) Probability distribution $P\left(\Gamma_{k}\right)[k=$ $1,2, \ldots, 8$, corresponding to Eqs. (8)-(15)] of the lowest-energy state with respect to the eigenspace of the Hamiltonian without hopping. (b) Probability distribution of the total atomic excitation number $N_{A}$. Parameters for both subfigures are $\Delta=0$ and $h=\lambda / 200$.

lowest-energy state contains two excitations on each site and the state $\left|2_{j}^{-}\right\rangle$is a maximally entangled state of the atom and the field on the jth site. Fig. 3(b) shows that in the lowestenergy state the components with both atoms in the ground state and both in the excited state are equally populated, which corresponds to a polaritonic insulator state for exact resonance and small hopping [20].

When $h=0$, the energy gap $E_{\Delta_{1,2}}$ goes to zero in the limit $\Delta / \lambda \rightarrow \pm \infty$, meaning the lowest level of the system is degenerate. However, a small but nonzero hopping value $(h / \lambda<<1)$ breaks this degeneracy, leading to a unique state with the lowest-energy. This lowest-energy state involves a superposition of polaritonic states, as seen in Fig. 4(a) and (c). The eigenstates of each independent site vary with the sign of the detuning, as seen in Fig. 4(b) and (d). For $\Delta / \lambda \rightarrow \infty$, the lowest-energy state is approximately $\left|g_{1} g_{2}\right\rangle \otimes\left[\frac{\sqrt{10}}{5}\left|2_{1} 2_{2}\right\rangle+\frac{1}{2}\right.$ $\left.\left(\left|1_{1} 3_{2}\right\rangle+\left|3_{1} 1_{2}\right\rangle\right)-\frac{\sqrt{5}}{10}\left(\left|4_{1} 0_{2}\right\rangle+\left|0_{1} 4_{2}\right\rangle\right)\right]$, which is a delocalized photon state (photonic superfluid state). For $\Delta / \lambda \rightarrow-\infty$, the lowest-energy state approximates $\left|e_{1} e_{2}\right\rangle \otimes\left[\frac{\sqrt{2}}{2}\left|1_{1} 1_{2}\right\rangle+\frac{1}{2}(\right.$ $\left.\left.\left|0_{1} 2_{2}\right\rangle+\left|2_{1} 0_{2}\right\rangle\right)\right]$, which is a coexisting state with the characteristics of both photonic superfluid and atomic insulator. The near-unity photon number in Fig. 4(d) is very different from the system with total excitation number $N=2$ investigated in Ref. [20] in which the lowest energy state is an atomic insulator state for small hopping and large negative detuning. This result has a simple physical explanation. When $\Delta$ is negative, the energy of the atomic excitation is lower than that of the photon. To ensure the energy to be minimum, two excitations should first be occupied by the atoms. The remaining two excitations are populated in the photonic modes, with the distribution being determined by the competition of the non-linear Kerr effect induced by the dispersive atom-cavity interaction and photon hopping.

Therefore, when the hopping is small, the lowest-energy state of the system undergoes quantum phase transitions from a polaritonic insulator state near exact resonance to a photonic superfluid state at large positive detuning.

In order to identify the polaritonic superfluid phase in the lowest energy state, we take the variance of the excitation number of the first atom $\Delta N_{1 A}$ as a measure, where $\Delta \hat{N}_{1 A}=$ $\left|e_{1}\right\rangle\left\langle e_{1}\right|$; the results are plotted in Fig. 5(a) and (c). $\Delta N_{1 A}$ is zero for the atomic insulator state, but is nonzero for a state with polaritonic characteristics. There are two regions with 

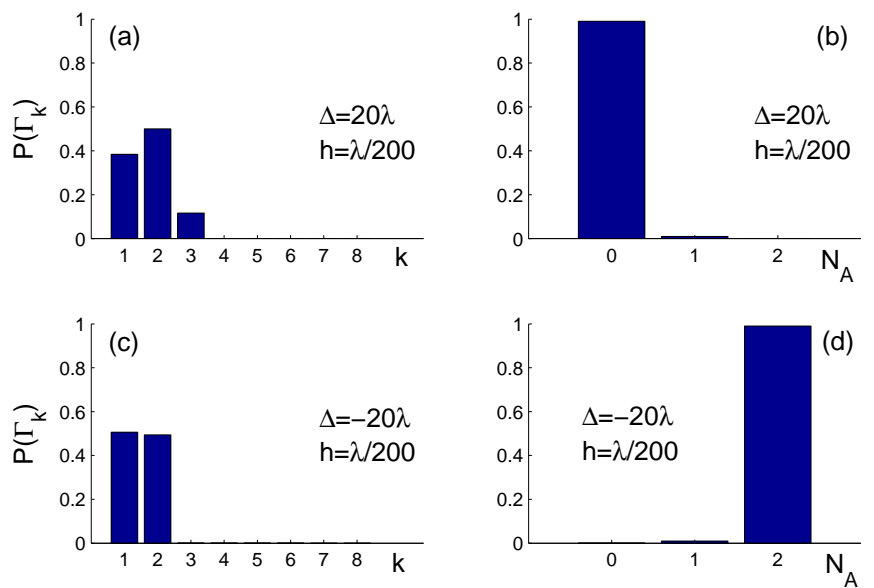

FIG. 4: (Color online) [(a)-(c)] Probability distribution $P\left(\Gamma_{k}\right)[k=$ $1,2, \ldots, 8$, corresponding to Eqs. (8)-(15)] of the lowest-energy state with respect to the eigenspace of the Hamiltonian without hopping. [(b)-(d)] Probability distribution of the total atomic excitation number $N_{A}$.
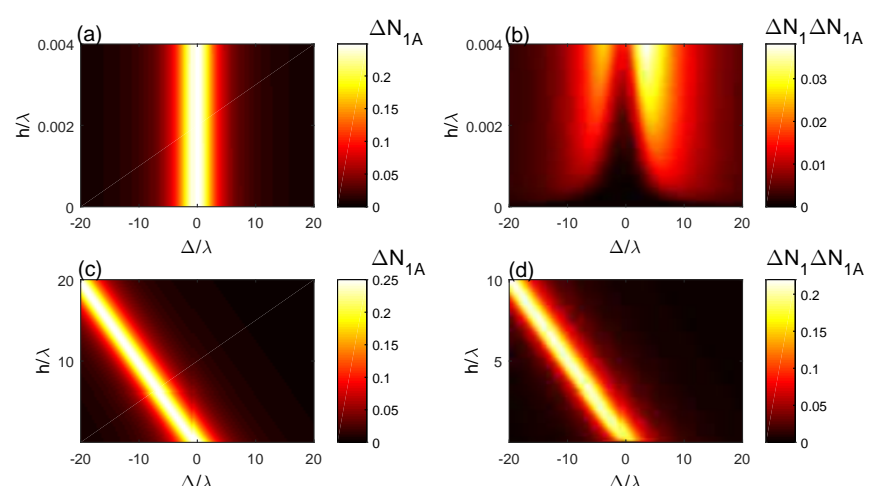

FIG. 5: (Color online) When the system is in the lowest-energy state and N=4: [(a)-(c)] $\Delta N_{1 A}$ as a function of $\Delta$ and $h$. [(b)-(d)] Product $\Delta N_{1} \Delta N_{1 A}$ as a function of $\Delta$ and $h$.

$\Delta N_{1 A}=0$ in Fig. 5(a): The region with $-\Delta>>\lambda$ is a coexisting state where two excitations are occupied by the atoms and localized at different sites, while the region with $\Delta>>\lambda$ is the photonic superfluid state where no atom is excited.

The product $\Delta N_{1} \Delta N_{1 A}$ may be used to characterize the polaritonic superfluid state as shown in Fig. 5(b), (c), and (d). $\Delta N_{1} \Delta N_{1 A}$ is zero for the polaritonic insulator state, but is nonzero for the polaritonic superfluid state. It is apparent that the polaritonic superfluid state appears in the near-resonance region for small hopping.

\section{Small atom-field interaction.}

In this section, when $\lambda<<h$, the atom-field interaction in $H$ is taken as a perturbation in order to analyze the system in the large-hopping regime.

When $h<-\Delta$, the lowest eigenenergy is $4 w_{c}+2 \Delta-2 h$, and the corresponding eigenstate is the coexisting state $\left|\varphi_{c o}\right\rangle$
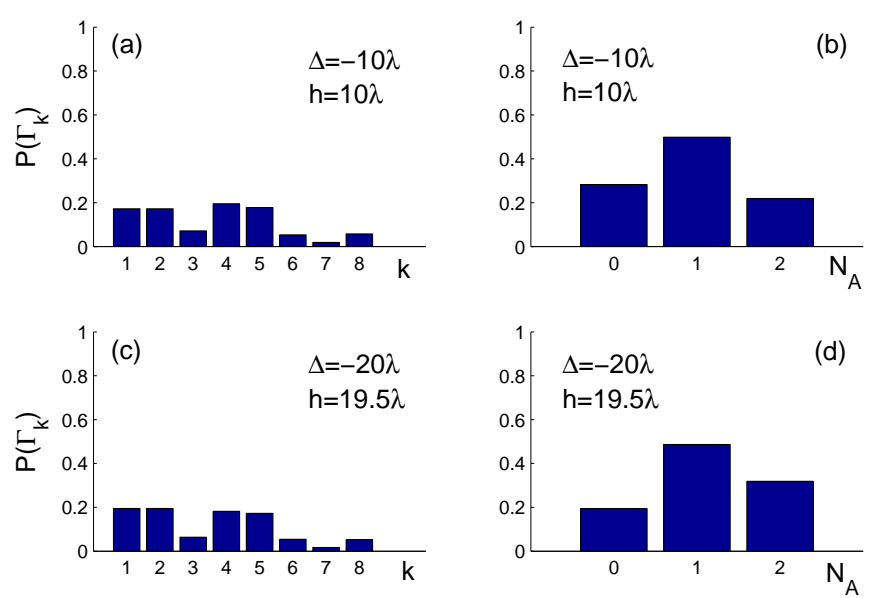

FIG. 6: (Color online) [(a)-(c)] Probability distribution $P\left(\Gamma_{k}\right)[k=$ $1,2, \ldots, 8$, corresponding to Eqs. (8)-(15)] of the lowest-energy state with respect to the eigenspace of the Hamiltonian without hopping. [(b)-(d)] Probability distribution of the total atomic excitation number $N_{A}$.

$=\left|e_{1} e_{2}\right\rangle \otimes\left[\frac{\sqrt{2}}{2}\left|1_{1} 1_{2}\right\rangle-\frac{1}{2}\left(\left|0_{1} 2_{2}\right\rangle+\left|2_{1} 0_{2}\right\rangle\right)\right]$. This coexisting state is similar to that in the regime $\Delta / \lambda \rightarrow-\infty$ for small hopping investigated in Sec. II.A., but is very different from the case of $N=2$ discussed in Ref. [20] in which the lowestenergy state is still an atomic insulator state for large negative detuning. For $h>-\Delta$ the lowest eigenenergy is $4 w_{c}-4 h$, and the corresponding eigenstate is the photonic superfluid state $\left|\varphi_{p s}\right\rangle=\left|g_{1} g_{2}\right\rangle \otimes\left[\frac{1}{4}\left(\left|4_{1} 0_{2}\right\rangle+\left|0_{1} 4_{2}\right\rangle\right)-\frac{1}{2}\left(\left|1_{1} 3_{2}\right\rangle+\left|3_{1} 1_{2}\right\rangle\right)+\right.$ $\left.\frac{\sqrt{6}}{4}\left|2{ }_{1} 2_{2}\right\rangle\right]$

When $h=-\Delta$ and $\lambda=0$, the lowest level of energy $4 w_{c}-2 h$ exhibits four-fold degeneracy with the eigenstates $\left|\varphi_{c o}\right\rangle,\left|\varphi_{p s}\right\rangle$, $\left|\varphi_{1}\right\rangle$, and $\left|\varphi_{2}\right\rangle$, where

$$
\begin{aligned}
\left|\varphi_{1}\right\rangle= & \frac{2 \sqrt{2}}{3}\left|e_{1} g_{2}\right\rangle \otimes\left[\left(\left|3_{1} 0_{2}\right\rangle-\left|0_{1} 3_{2}\right\rangle\right)\right. \\
& \left.-\sqrt{3}\left(\left|2_{1} 1_{2}\right\rangle-\left|1_{1} 2_{2}\right\rangle\right)\right], \\
\left|\varphi_{2}\right\rangle= & \frac{2 \sqrt{2}}{3}\left|g_{1} e_{2}\right\rangle \otimes\left[\left(\left|3_{1} 0_{2}\right\rangle-\left|0_{1} 3_{2}\right\rangle\right)\right. \\
& \left.-\sqrt{3}\left(\left|2_{1} 1_{2}\right\rangle-\left|1_{1} 2_{2}\right\rangle\right)\right] .
\end{aligned}
$$

The small atom-field interaction breaks this four-fold degeneracy, leading to a unique state with the lowest-energy. As shown in Fig. 5(d), for large hopping this eigenstate can be identified as a polaritonic superfluid state when $h \simeq-\Delta$. The probability distributions of such two eigenstates with respect to the eigenspace $\Gamma_{k}$ and atomic excitation number $N_{A}$ are plotted in Fig. 6. In this case all the polariton subspaces are occupied and atoms are partially excited, indicating that the superfluid state has polaritonic characteristics.

\section{Total excitation number $N$.}

In this section, we examine the dependence of the variances $\Delta N_{1} / N, \Delta N_{1 A}$, and $\left(\Delta N_{1} / N\right) \Delta N_{1 A}$ on the total excitation number $N$ in the lowest-energy state with fixed hopping strength. 

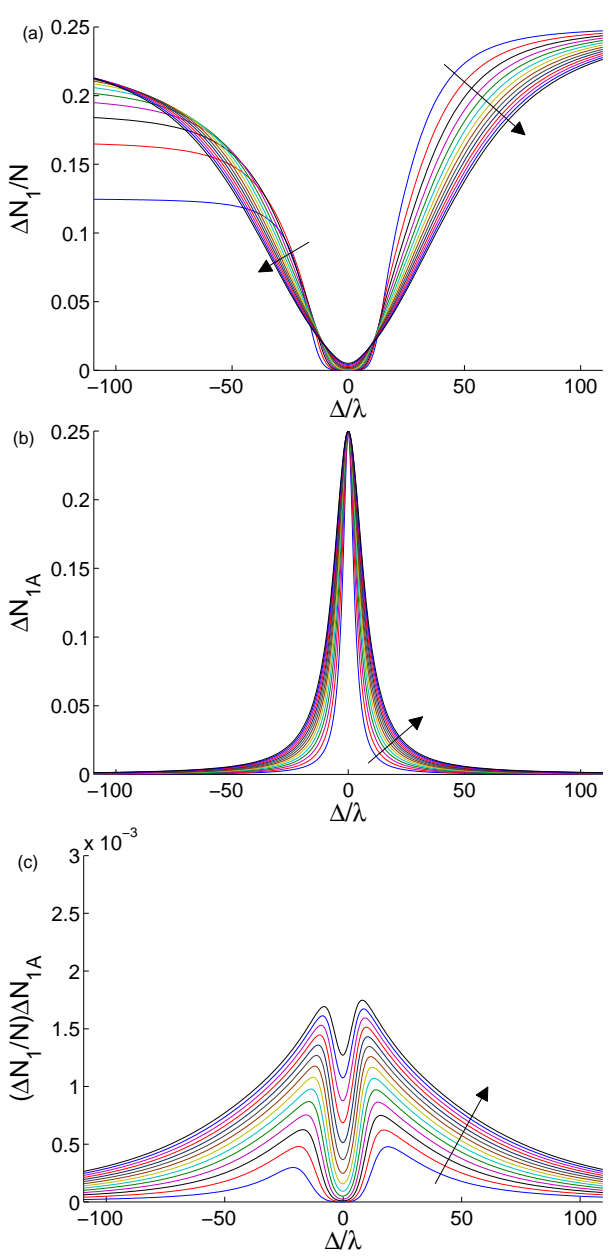

FIG. 7: (Color online) Excitation number variance as a function of the detuning $\Delta$ in the lowest-energy state of the $N$-excitation $(N=4,6,8, \ldots, 30)$ coupled atom-cavity system when $h=10^{-4} \lambda$, where different excitation number variances are: (a) $\Delta N_{1} / N$; (b) $\Delta N_{1 A}$; (c) $\left(\Delta N_{1} / N\right) \Delta N_{1 A}$. The direction of the black arrows represents the increasing trend for $N$ in these subfigures.

Here, the relative total excitation number variance $\Delta N_{1} / N$ is used to eliminate the effect of simply expanding the total excitation number and characterize the polaritonic superfluid.

\section{Small hopping.}

In Fig. 7, we plot different excitation number variances as functions of the detuning in the lowest-energy state of the $N$-excitation coupled atom-cavity system under the smallhopping situation, where $N=4,6,8, \ldots, 30$.

Figure 7(a) shows the effect of increasing $N$ on the relative total excitation number variance $\Delta N_{1} / N$. As before, $\Delta N_{1} / N>0$ indicates a delocalized or superfluidlike state, while $\Delta N_{1} / N \sim 0$ indicates a localized, insulatorlike state. For large positive detuning, $\Delta N_{1} / N>0$ corresponds to the photonic superfluid state, while for large negative detuning, $\Delta N_{1} / N>0$ corresponds to the coexisting state with characteristics of both photonic superfluid and atomic insulator. In the limit of very large $|\Delta|$, as $N$ increases, the relative total excitation number variance decreases, and the transition from the insulator to superfluid becomes slower. Figure 7(a) also shows that the region over which $\Delta N_{1} / N \sim 0$, indicating an insulator state, narrows as $N$ increases. The photon hopping strength is set to be $h=10^{-4} \lambda$ similar to that of Sec. III.A. For arbitrary even $N>2$ the gap between the lowest two states for $h=0$ becomes $(2 \sqrt{N}-\sqrt{N-1}-\sqrt{N+1}) \lambda$, which goes to zero as $N \rightarrow \infty$. Therefore as $N$ increases the photon blockade, which leads to the polaritonic insulator state, weakens and hence can be easily overcome by the photon hopping. When $N \geq 20$, the system can not stay in the insulator state for $h=10^{-4} \lambda$.

Figure 7(b) shows the atomic excitation number variance $\Delta N_{1 A}$ as a function of $\Delta / \lambda$ for different $N$. As $N$ increases, the maximum value of $\Delta N_{1 A}$ remains unchanged, but the region with nonzero $\Delta N_{1 A}$ is broadened. The maximum variance of the atomic excitation number depends on the number of atoms in the system, which is independent of the excitation number $N$. However, the on-resonance Rabi frequency scales as $\sqrt{N} \lambda$. Therefore the range of detunings over which the atom-field interaction is large enough to produce polaritonic behavior increases with $N$.

The combined effect of these features is shown in Fig. 7(c), where the product of the two variances as a function of $\Delta / \lambda$ is plotted. Here a nonzero value corresponds directly to a polaritonic superfluid state. It is clear that the polaritonic superfluid region extends over a wider range of $\Delta$ values as $N$ increases. The dip at $\Delta=0$ that indicates the transition to a polaritonic insulator state fails to go to 0 when $N \geq 20$, because, as discussed above, the value of $h$ used in this plot is large enough to overcome the energy gap between the lowest two levels produced by the atom-cavity interaction when $N \geq 20$.

\section{Small atom-field interaction.}

In Fig. 8, we plot various excitation number variances as functions of the detuning for the lowest-energy state of the $N$-excitation coupled atom-cavity system, where $N=$ $4,6,8, \ldots, 30$, in the small atom-field interaction regime.

Figure 8(a) shows that, in the region $\Delta \leq-h$, the relative total excitation number variance $\Delta N_{1} / N$ becomes larger as $N$ increases, indicating that the superfluid is enhanced. This is due to the fact that when $\Delta$ passes the critical point $\Delta_{c}=-h$, the values of $\Delta N_{1} / N$ all converge to the maximum $1 / 4$. Figure 8 (b) shows the atomic excitation number variance $\Delta N_{1 A}$ as a function of $\Delta$ for different $N$ when $h=50 \lambda$. The result is similar to that of Fig. 7(b), except that the nonzero region indicating that polaritonic states is now centered around $\Delta=$ $-h$. The product of the two variances as a function of $\Delta / \lambda$, plotted in Fig. 8(c), demonstrates the existence of a distinct polaritonic superfluid state in the vicinity of $\Delta=-h$. As $N$ increases, the width of the polaritonic superfluid region also increases. The maximum value of the variance product also increases with $N$. These features suggest that, in the large hopping limit, the polaritonic superfluid state may be easier to observe as the total excitation number increases. 

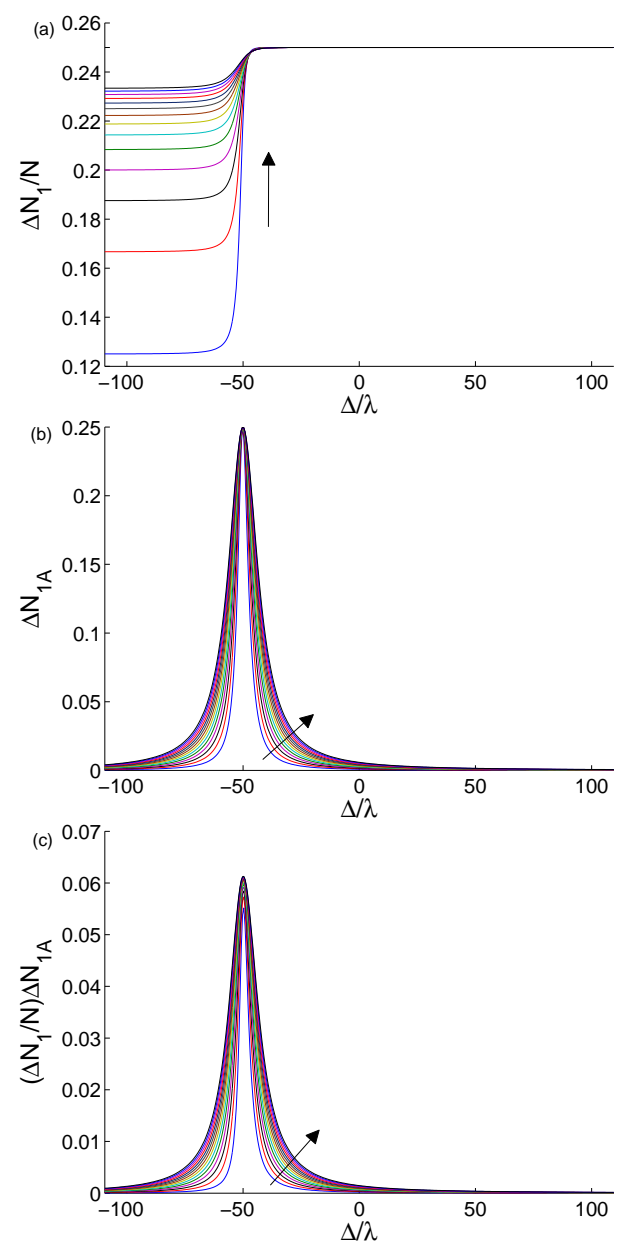

FIG. 8: (Color online) Excitation number variance as a function of the detuning $\Delta$ in the lowest-energy state of the $N$-excitation $(N=4,6,8, \ldots, 30)$ coupled atom-cavity system when $h=50 \lambda$, where different excitation number variances are: (a) $\Delta N_{1} / N$; (b) $\Delta N_{1 A}$; (c) $\left(\Delta N_{1} / N\right) \Delta N_{1 A}$. The direction of the black arrows represents the increasing trend for $N$ in these subfigures.

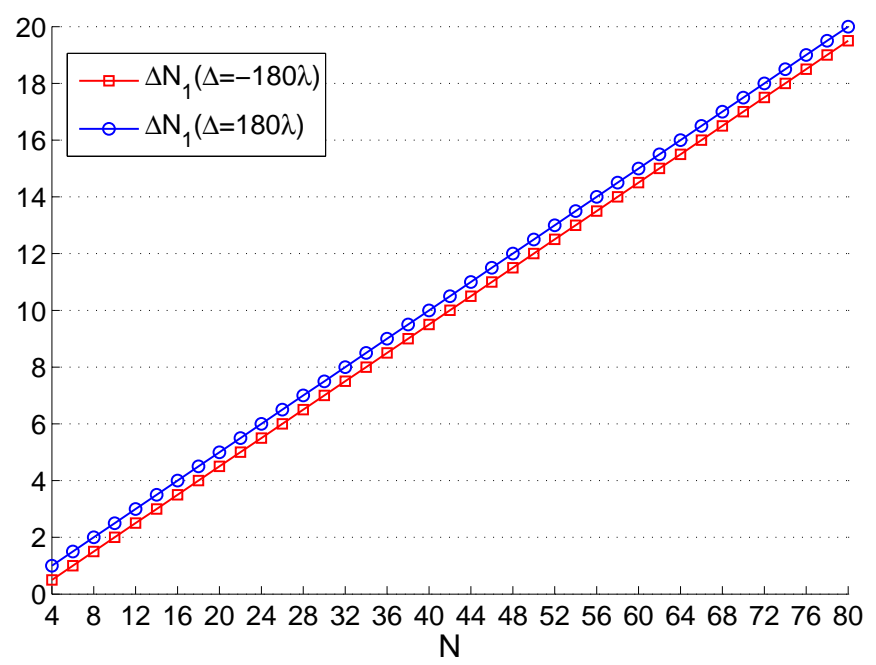

FIG. 9: (Color online) $\Delta N_{1}$ as a function of $N$ in the lowest energy state for the large-detuning limits, where $h=25 \lambda$.

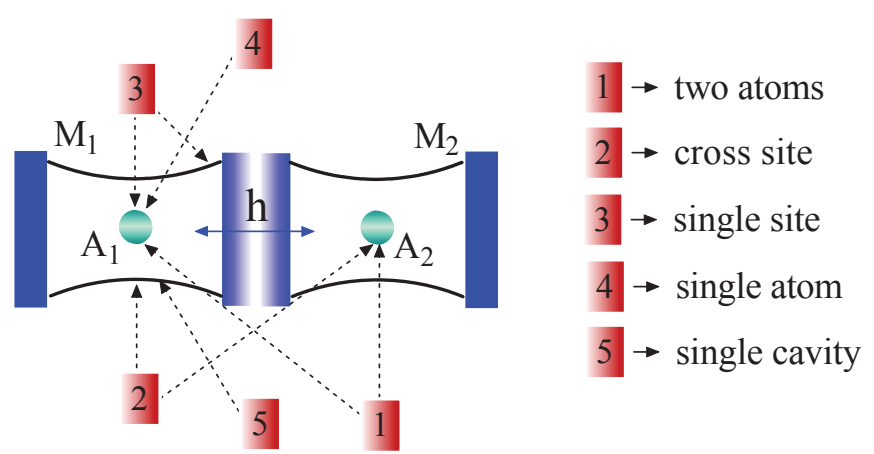

FIG. 10: (Color online) Classification for five bipartitions in the multi-excitation coupled array.

In Fig. 9, we plot the variance of the total excitation number in the first site $\Delta N_{1}$ as a function of the total excitation number for $\Delta \rightarrow-\infty$ and $\Delta \rightarrow \infty$. The results show that $\Delta N_{1}$ has a linear dependence on $N$ in both limits.

\section{Nonclassical properties.}

\section{Entropy.}

Since the lowest-energy state of the two-site system is a pure state, we can use the von Neumann entropy to measure the entanglement of its lowest-energy state. For a composite system $X Y$, the entropy $S$ can be calculated through the density matrix $\rho_{X Y}$. For example, the entropy $S\left(\rho_{X}\right)=$ $-\operatorname{Tr}\left[\rho_{X} \log _{2}\left(\rho_{X}\right)\right]$ based on the reduced density matrix $\rho_{X}=$ $\operatorname{Tr}_{Y}\left[\rho_{X Y}\right]$ measures the entanglement between subsystem $X$ and subsystem $Y$ of the composite system $X Y$.

In the following, we first focus on the analysis for the entanglement in the lowest-energy state of the two-site system with $N=4$, in which the entropy's behavior maps the quantum phase transition of polaritonic excitations in Fig. 2 and Fig. 5, and later generalize it to the case with $N>4$.

For two identical sites, we define five bipartitions to resolve the entanglement in the lowest-energy state, as classified in Fig. 10, each of which represents one kind of entanglement measure. $S\left(\rho_{A 1 M 1}\right)$ represents the single-site entropy. $S\left(\rho_{A 1}\right)$ and $S\left(\rho_{M 1}\right)$ represent the entanglement of a single atom and a single cavity within the first site, respectively. $S\left(\rho_{A 1 A 2}\right)$ and $S\left(\rho_{A 1 M 2}\right)$ represent the entanglement of two atoms and cross site, respectively.

Figure 11 shows the entropies of five bipartitions as a function of the detuning in the small-hopping limit when $N=4$. At large negative detuning, the entropies of the single atom and two atoms both drop to zero while the other three entropies asymptotically approach 1.5. In this case, the two atoms are both in the excited states, corresponding to the coexisting state involving the characteristics of both atomic insulator and photonic superfluid, which is very different from Ref. [21] where five entropies of an atomic insulator all approach zero. At resonance condition $(\Delta=0)$, four of these five entropies reach a local maximum, except for the entropy of 


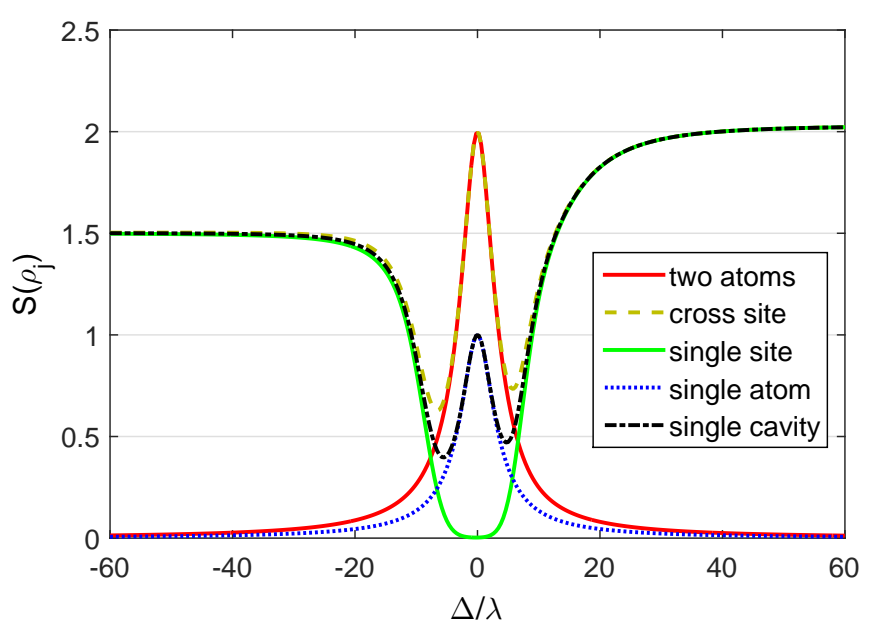

FIG. 11: (Color online) Entropy of five bipartitions as a function of $\Delta$ in the small-hopping limit for $h=5 \times 10^{-4} \lambda$.

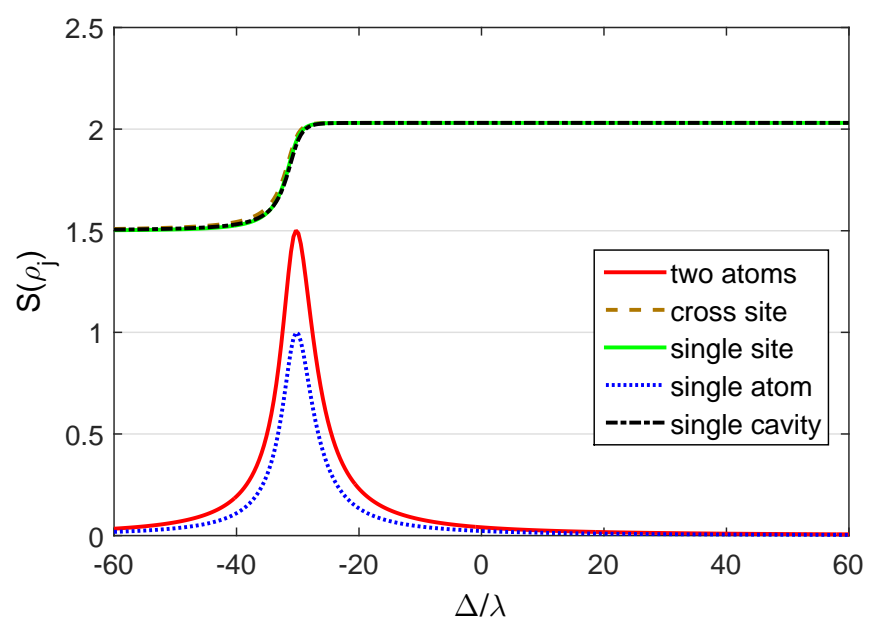

FIG. 12: (Color online) Entropy of five bipartitions as a function of $\Delta$ in the large-hopping limit for $h=30 \lambda$.

the single site being zero, indicating the polaritonic entanglement induced by the resonant Jaynes-Cummings interaction between the atom and the local field mode. For large positive detuning, similar to the entropy's behaviors of large negative detuning, the entropies of the single atom and two atoms both drop to zero but the other three entropies asymptotically approach 2. In this region, the atoms are both in the ground states, corresponding to the photonic superfluid state. These entropies are higher than those in Ref. [21] where those corresponding entropies asymptotically approach 1.5 , which is due to more entangled photon-state components in the photonic superfluid state of the four-excitation coupled array than that of the two-excitation coupled array.

We remark that although the measure of a genuine multipartite entanglement is still absent, however, if all bipartitions of the system are all entangled, the system undoubtedly exhibits the genuine multipartite entanglement.

In Fig. 12, we plot the entropies of five bipartitions as a function of the detuning in the large-hopping limit when
$N=4$. The system at large negative detuning is a coexisting state involving the characteristics of both atomic insulator and photonic superfluid, while at large positive detuning is a photonic superfluid state, which is similar to the result of the case with small hopping. We find that the entropies of five bipartitions all reach a local maximum near the critical point $h=-\Delta$, demonstrating that a genuine 4-body entanglement appears in the polaritonic superfluid state. In Fig. 11, the entropies of single cavity and cross site for the small hopping have two minimum, which is different behavior from those in the large hopping case in Fig. 12. This is because that for $\Delta=0$ and $h / \lambda<<1$ in Fig. 11, the lowest-energy state is a maximally entangled state of the atom and the field in each site, which corresponds to one maximum and two minimum in entropies of single cavity and cross site. However, for $h / \lambda>>1$ in Fig. 12 , the lowest-energy state continuously varies from a coexisting state at large negative detuning to a photonic superfluid state at large positive, without becoming maximally entangled state of the atom and the field in $\Delta=0$.

Although only two extreme situations including the smalland large-hopping limits are analyzed here, the entropy curves of five bipartitions vary between the two extreme situations with a predictable behavior when $N=4$ which we do not plot here.

In Fig. 13, we plot the entropies of five bipartitions as a function of $\Delta / \lambda$ for both the small-hopping limit and largehopping limit $(N=8,12)$. It is predictable from the results of Fig. 13 that as $N$ increases the entropies of two atoms and the single atom keep invariant when $\Delta=0$ and $\Delta \rightarrow \pm \infty$, while the other three entropies reach the same stable value when $\Delta \rightarrow \pm \infty$. As plotted in Fig. 14, the entropy describing the entanglement of the photonic superfluid state has a quadratic-like dependence on the increasing $N$ in the largedetuning limit, where the atoms are both in the excited states for $\Delta \rightarrow-\infty$ and both in the ground states for $\Delta \rightarrow \infty$. We use an optimizing power-fit algorithm to plot the fitting curves for the numerical solution in Fig. 14, and find approximately analytical expressions between $S\left(\rho_{2,3,5}\right)$ and $N$ as:

$$
S\left(\rho_{2,3,5}\right) \propto N^{-1 / 5}
$$

and

$$
S\left(\rho_{2,3,5}\right) \propto N^{-1 / 100}
$$

for Fig. 14(a) and 14(b), respectively. This result demonstrates that the entanglement of photons in the photonic superfluid state has an approximately quadratic-like dependence on the total excitation number $N$ when the whole system is in the large-detuning limit.

The behavior of the lowest-energy state's entropy reveal$\mathrm{s}$ the entanglement among atoms and photons, and provides another intuitive approach for discriminating different regions of the phase diagram. In the polaritonic insulator state, polaritonic entanglement between the atom and photons at each site almost remains the same even when the total excitation number increases. In the coexisting state and photonic superfluid state, the bipartite entanglement between two sites becomes larger as the total excitation number increases. Especially in 

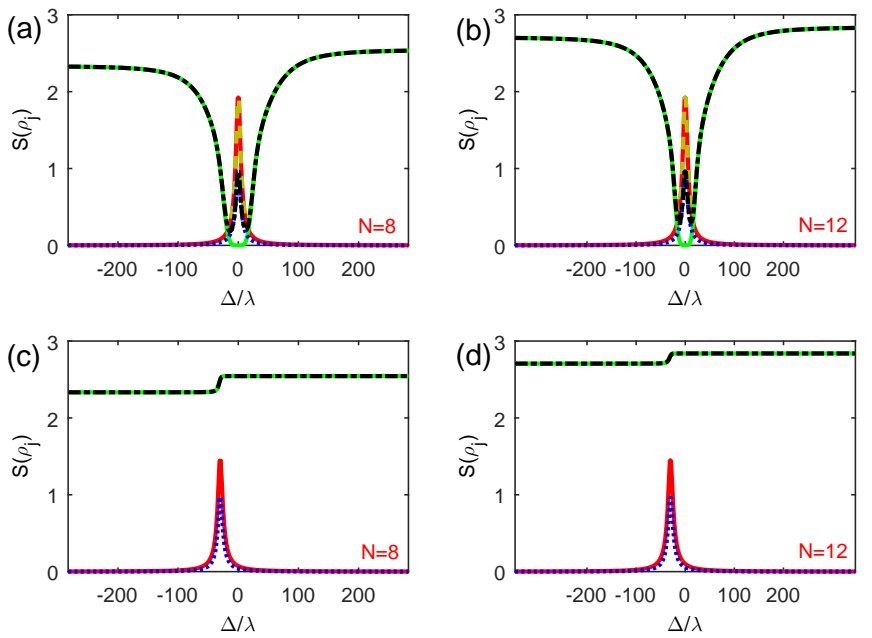

FIG. 13: (Color online) Entropy of five bipartitions as a function of $\Delta$. In the small-hopping limit: [(a)-(b)] $h=10^{-5} \lambda$. In the largehopping limit: [(c)-(d)] $h=30 \lambda$. The concrete representation of each line shape is the same as that in Fig. 1.
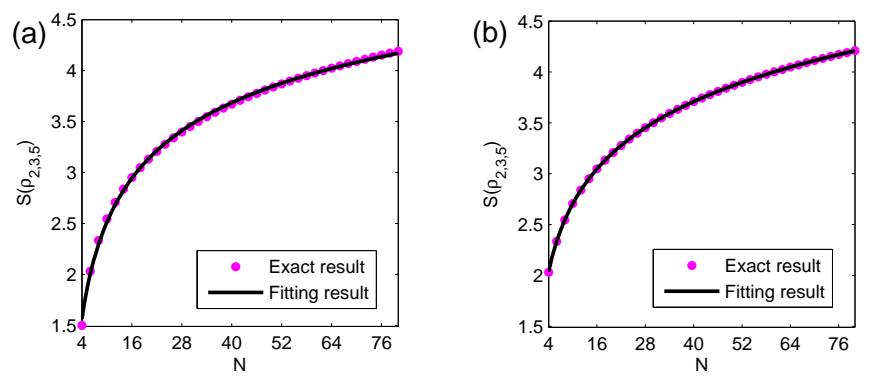

FIG. 14: (Color online) Three of five bipartitions as functions of the total excitation number $N(N \geq 4)$ when the system is in the large-detuning limit, i.e., $S_{\rho_{2}}, S_{\rho_{3}}$, and $S_{\rho_{5}}$ respectively represent the entropy of cross site, single site, and single cavity: (a) $\Delta / \lambda \rightarrow-\infty$; (b) $\Delta / \lambda \rightarrow \infty$. The fitting curves are mathematically plotted based on an optimizing power-fit algorithm.

the polaritonic superfluid state, the genuine multipartite entanglement appears but the entanglements of the single atom and two atoms almost keep invariant as the total excitation number increases. It is interesting to see that the genuine multipartite entanglement in the polaritonic superfluid state for large hopping is more obvious than that in the case with only two excitations, especially the entropies (of single site, cross site, and single cavity) increase with the increasing $N$, meaning that the degree of the multipartite entanglement of the polaritonic superfluid state for this multi-excitation system is much bigger compared with that in Ref. [21]. The entropies of five bipartitions should be taken together to provide a full description for the entanglement of the lowest-energy state.
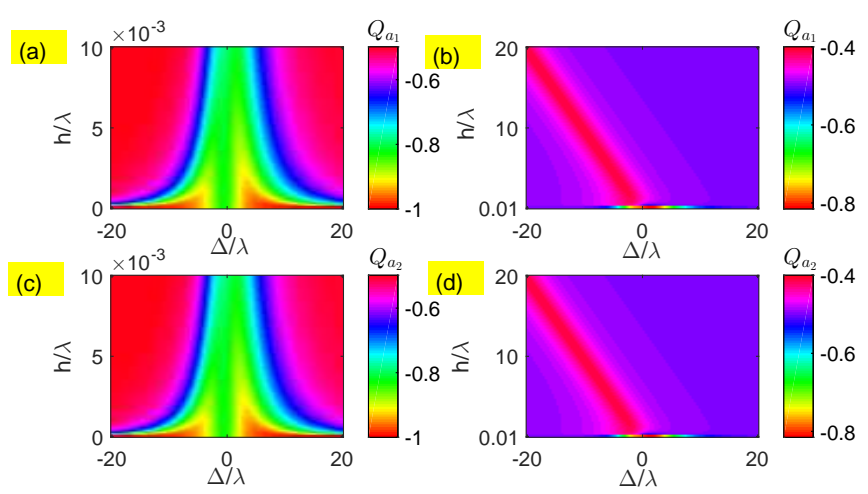

FIG. 15: (Color online) $Q_{k}$ as a function of $\Delta$ and $h$ for the lowestenergy state of the system when $N=4$. [(a)-(b)]: $k=a_{1}$; [(c)-(d)]: $k=a_{2}$.

\section{Mandel Q-parameters.}

We calculate the Mandel Q-parameters defined for $a_{1}$ and $a_{2}$ as:

$$
\begin{aligned}
Q_{a_{1}} & =\frac{\left\langle a_{1}^{\dagger} a_{1} a_{1}^{\dagger} a_{1}\right\rangle-\left\langle a_{1}^{\dagger} a_{1}\right\rangle^{2}-\left\langle a_{1}^{\dagger} a_{1}\right\rangle}{\left\langle a_{1}^{\dagger} a_{1}\right\rangle}, \\
Q_{a_{2}} & =\frac{\left\langle a_{2}^{\dagger} a_{2} a_{2}^{\dagger} a_{2}\right\rangle-\left\langle a_{2}^{\dagger} a_{2}\right\rangle^{2}-\left\langle a_{2}^{\dagger} a_{2}\right\rangle}{\left\langle a_{2}^{\dagger} a_{2}\right\rangle} .
\end{aligned}
$$

If $-1 \leq Q_{k}<0\left(k=a_{1}, a_{2}\right)$, the mode $k$ exhibits sub-Poisson statistics. As shown in Fig. 15, we plot the parameter $Q_{k}$ as a function of $\Delta$ and $h$ for the lowest-energy state of the system when $N=4$. The result shows that both modes $a_{1}$ and $a_{2}$ exhibit similar sub-Poisson statistics in the lowest-energy state of the system. It is apparent that the value of $Q_{k}$ in insulator state is much smaller than that in superfluid state, which may be another useful indicator for distinguishing the regions with the characteristic of insulator and superfluid in the corresponding phase diagram of Fig. 2 and Fig. 5. The value of $Q_{k}$ in the coexisting state is very close to that in the photonic superfluid state, meaning that the coexisting state and photonic superfluid state exhibit similar sub-Poisson statistics. Interestingly, the minimum value of $Q_{k}$ appears in the polaritonic superfluid state, while its maximum appears in the polaritonic insulator state.

In Fig. 16, we plot the parameter $Q_{a_{1}}$ as a function of the detuning in the lowest-energy state of the $N$-excitation coupled atom-cavity system under the small-hopping situation in Fig. 16(a) and large-hopping situation in Fig. 16(b), where $N=4,6,8, \ldots, 30$ and the behavior of $Q_{a_{2}}$ is very similar to that of $Q_{a_{1}}$ here. In both situations, $Q_{a_{1}}$ is independent of $N$ and approaches to -0.5 for both large negative and positive detunings. This can be explained from the approximately analytical lowest-energy state $\left|\psi_{g, N}^{p h}\right\rangle=\frac{1}{\sqrt{N !}}\left[\frac{1}{\sqrt{2}}\left(a_{1}^{\dagger}-a_{2}^{\dagger}\right)\right]^{N}\left|0_{1}\right\rangle\left|0_{2}\right\rangle$ derived in the method section, where $Q_{a_{1}}=Q_{a_{2}}=-0.5$ is obtained for the state $\left|\psi_{g, N}^{p h}\right\rangle$. For $\Delta \simeq 0$ in the small-hopping situation or $h \simeq-\Delta$ in the large-hopping situation, $Q_{a_{1}}$ becomes smaller as $N$ increases, indicating that the sub-Poisson 

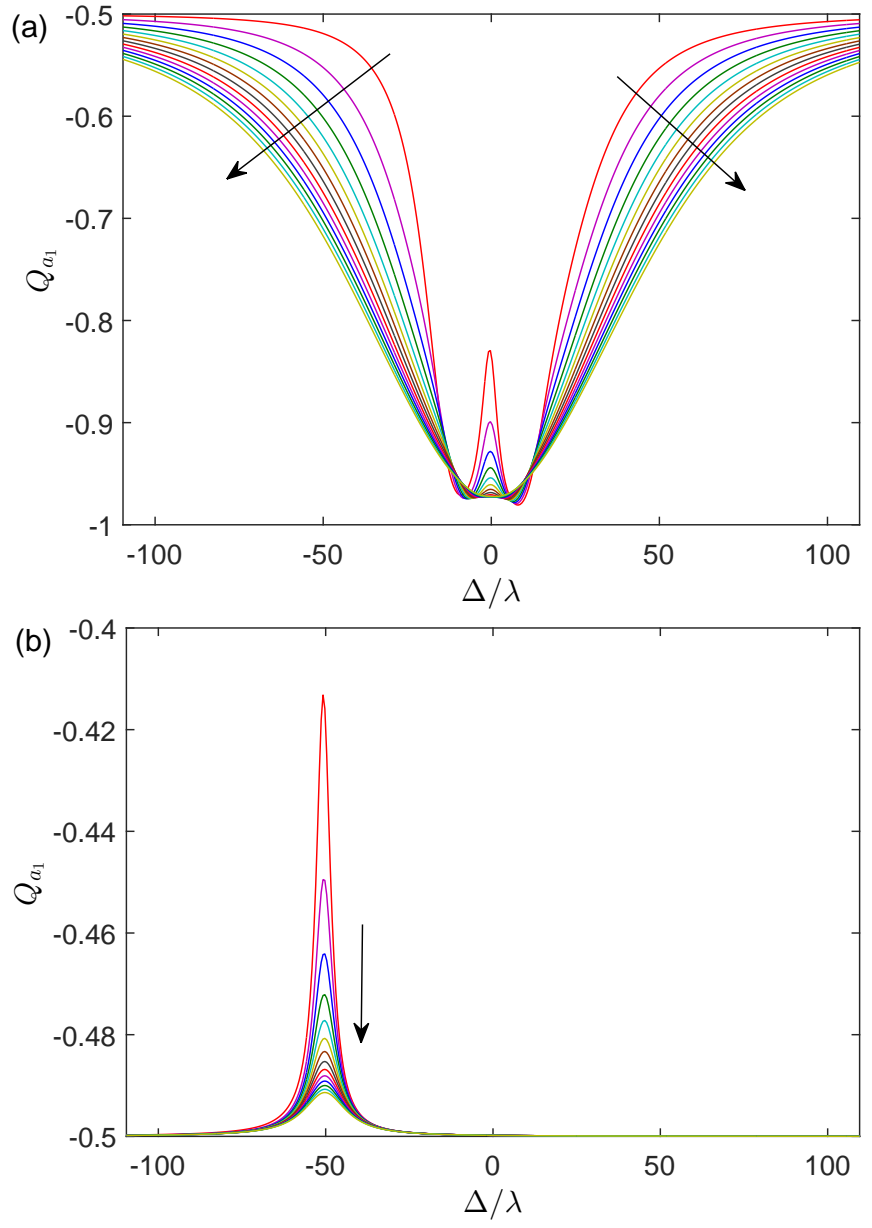

FIG. 16: (Color online) $Q_{a_{1}}$ as a function of the detuning $\Delta$ in the lowest-energy state of the $N$-excitation $(N=4,6,8, \ldots, 30)$ coupled atom-cavity system for two situations: (a) $h=10^{-4} \lambda$; (b) $h=50 \lambda$. The direction of the black arrows represents the increasing trend for $N$ in these subfigures.

statistics of two field modes are more obvious as the total excitation number increases.

\section{Second-order cross-correlation function.}

We also consider the second-order cross-correlation function $Q_{a_{1} a_{2}}$, which is defined as:

$$
Q_{a_{1} a_{2}}=\frac{\left\langle a_{1}^{\dagger} a_{1} a_{2}^{\dagger} a_{2}\right\rangle}{\left\langle a_{1}^{\dagger} a_{1}\right\rangle\left\langle a_{2}^{\dagger} a_{2}\right\rangle}-1
$$

$Q_{a_{1} a_{2}}$ is zero for uncorrelated states, positive for correlated states, and negative for anticorrelated states. As shown in Fig. 17, we plot the parameter $Q_{a_{1} a_{2}}$ as a function of $\Delta$ and $h$ for the lowest-energy state of the system when $N=4$. Interestingly, we find that the insulator states can also be identified as uncorrelated states but the superfluid states are always anticorrelated states. The value of $Q_{a_{1} a_{2}}$ in the coexisting state is obviously smaller than that in the photonic superfluid state,
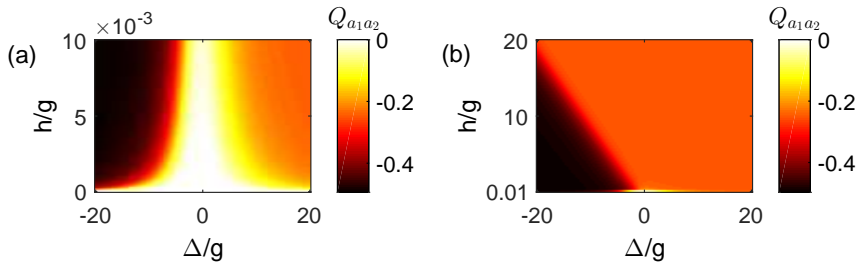

FIG. 17: (Color online) [(a)-(b)] $Q_{a_{1} a_{2}}$ as a function of $\Delta$ and $h$ for the lowest-energy state of the system when $N=4$.

which can be explained from the fact that in the coexisting state $(\Delta \rightarrow-\infty)$ two excitations are occupied by the atoms while in the photonic superfluid state $(\Delta \rightarrow \infty)$ no atom is excited, inducing stronger cross correlations between two field modes in the coexisting state.

In Fig. 18, we plot the parameter $Q_{a_{1} a_{2}}$ as a function of the detuning in the lowest-energy state of the $\mathrm{N}$-excitation coupled atom-cavity system under the small-hopping situation in Fig. 18(a) and large-hopping situation in Fig. 18(b), where $N=4,6,8, \ldots, 30$. For small-hopping situation, the value of $Q_{a_{1} a_{2}}$ keeps zero and is independent of $N$ within the nearresonance $(\Delta \simeq 0)$ regions. This is because that when $\Delta \simeq 0$ and $h<<\lambda$ the lowest-energy state of the system is a polaritonic insulator state which has no cross correlation between two field modes, corresponding to the uncorrelated states in Fig. 18(a). In both situations, when $\Delta \rightarrow \pm \infty, Q_{a_{1} a_{2}}$ increases quickly as $N$ increases from 4 to 16 , then increases much slowly and approaches to zero as $N$ further increases. This can be explained by calculating $Q_{a_{1} a_{2}}$ in both large-detuning limits. Within the large-detuning limits, the lowest-energy state of the system is approximately given by the state $\left|\psi_{g, N}^{p h}\right\rangle$ and we calculate $Q_{a_{1} a_{2}}$ for the state $\left|\psi_{g, N}^{p h}\right\rangle$ as follows:

$$
\begin{aligned}
& Q_{a_{1} a_{2}}=-(N-2)^{-1},(N \rightarrow-\infty) \\
& Q_{a_{1} a_{2}}=-N^{-1},(N \rightarrow \infty)
\end{aligned}
$$

which analytically shows that $Q_{a_{1} a_{2}}$ is inversely proportional to $N$ in both large-detuning limits and explaining the increasing behavior of $Q_{a_{1} a_{2}}$ in Fig. 18 for the increasing $N$. As $N$ further increases, the difference of $Q_{a_{1} a_{2}}$ between the large negative- and positive-detuning situations vanishes, i.e., $\lim _{N \rightarrow \pm \infty}\left|(N-2)^{-1}-N^{-1}\right| \simeq 0$, indicating that the effect caused by the atomic state on the anticorrelated property between two field modes largely weakens when $N$ is big enough.

\section{METHODS}

In the limit of large detuning, the two field modes effectively decouple from the atoms and can be approximately described by the interaction Hamiltonian:

$$
H_{p h}=w_{c}\left(a_{1}^{\dagger} a_{1}+a_{2}^{\dagger} a_{2}\right)+h\left(a_{1}^{\dagger} a_{2}+a_{2}^{\dagger} a_{1}\right)
$$



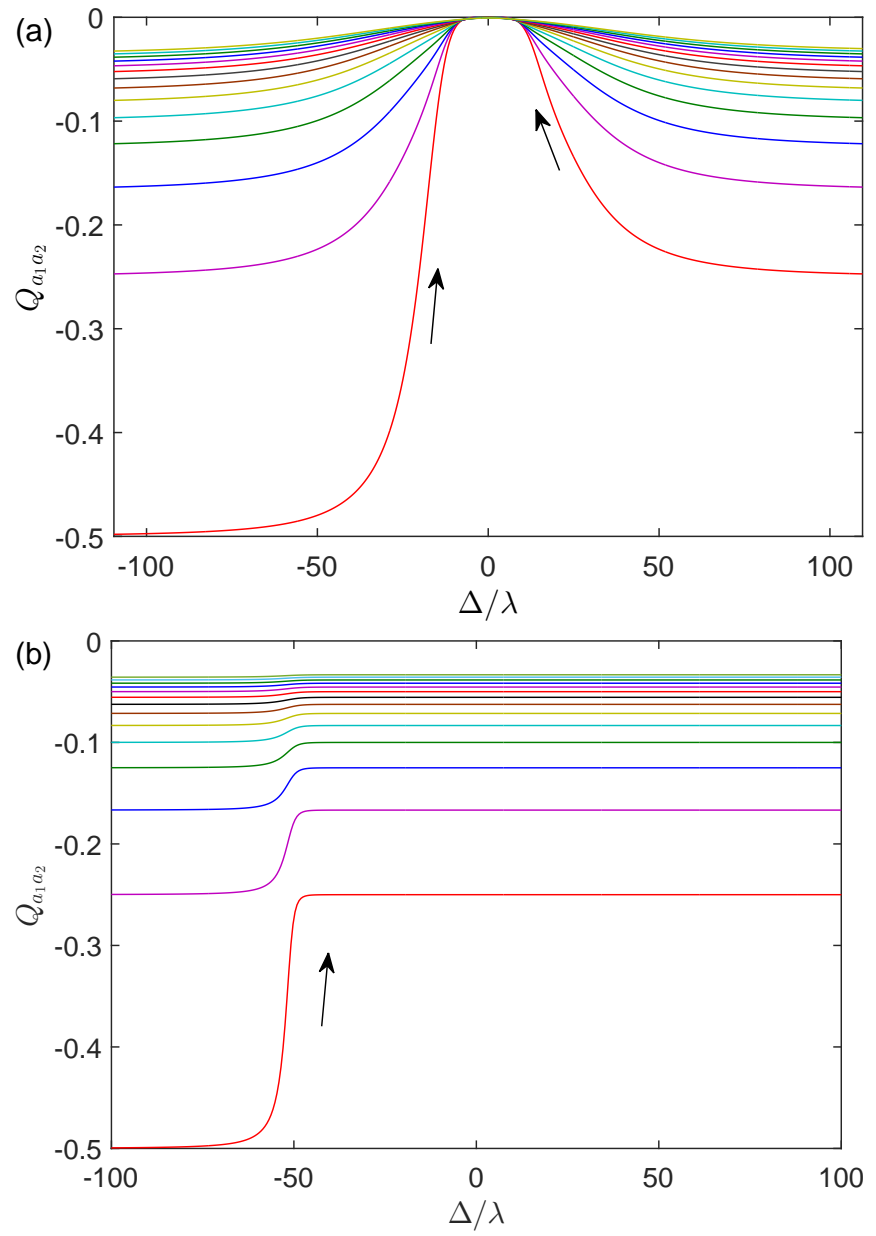

FIG. 18: (Color online) $Q_{a_{1} a_{2}}$ as a function of the detuning $\Delta$ in the lowestenergy state of the $N$-excitation $(N=4,6,8, \ldots, 30)$ coupled atom-cavity system for two situations: (a) $h=10^{-4} \lambda$; (b) $h=50 \lambda$. The direction of the black arrows represents the increasing trend for $N$ in these subfigures.

$H_{p h}$ is easily diagonalized by defining the delocalized mode operators:

$$
b_{ \pm}=\frac{1}{\sqrt{2}}\left(a_{1} \pm a_{2}\right),
$$

where $\left[b_{+}, b_{-}\right]=0$. In terms of these new operators, $H_{p h}$ becomes:

$$
H_{p h}=\left(w_{c}+h\right) b_{+}^{\dagger} b_{+}+\left(w_{c}-h\right) b_{-}^{\dagger} b_{-} .
$$

Assuming $h>0$, the lowest-energy state in its $N$-excitation subspace is given by:

$$
\left|\psi_{g, N}^{p h}\right\rangle=\frac{1}{\sqrt{N !}}\left(b_{-}^{\dagger}\right)^{N}\left|0_{1}\right\rangle\left|0_{2}\right\rangle .
$$

Thus, we straightforwardly calculate $\Delta N_{1}$ in the largedetuning limits:

$$
\Delta N_{1} \simeq \frac{(N-2)}{4},(\Delta \rightarrow-\infty)
$$

$$
\Delta N_{1} \simeq \frac{N}{4},(\Delta \rightarrow \infty)
$$

which analytically demonstrates the linear relationship between $\Delta N_{1}$ and $N$ in the large-detuning limit.

For large positive $\Delta$, the atoms are both in the ground states and so the lowest-energy state of the system is given by $\left|\psi_{g, N}^{p h}\right\rangle$ : all $N$ excitations go into the delocalized mode $b_{-}$. On the other hand, for large negative $\Delta$ the atoms are both excited, leaving only $N-2$ excitations in the field modes. In other words, in this regime the lowest-energy state is the combination of the atomic insulator state and the photonic superfluid state. The effect of the atomic insulator state on the total excitation number variance is weakened as $N$ increases. This behavior is evident in Fig. 7(a) and Fig. 8(a). The state $\left|\psi_{g, N}^{p h}\right\rangle$ has a larger number variance for large $N$ but is not in a sense any more delocalized, since the delocalized mode defined by $b_{-}$ is maximally delocalized over the two sites. Larger $N$ simply means there are more photons in the delocalized mode $b_{-}$, which explains the behavior shown in Fig. 8(a), where the curves for different $N$ all converge to the same constant.

\section{DISCUSSION}

We have investigated the quantum phase transition behavior of the lowest-energy state in a multi-excitation coupled atomcavity system. The case of total excitation number $N=4$ has been treated both analytically and numerically, and the results are then generalized to the case with higher excitation numbers. We have identified an interesting coexisting phase involving characteristics of both photonic superfluid and atomic insulator in the large negative detuning conditions, which does not appear for the two-excitation case and has not been previously discovered. We find that the region where the system exhibits the polaritonic characteristic becomes broader as the total excitation number increases. We demonstrate that $\Delta N_{1}$ has a linear dependence on $N$ in the large-detuning limit. we have alternatively used the order parameters of entropy, the Mandel Q-parameter, and the second-order cross-correlation function to reveal different nonclassical properties of the lowest-energy state in a multi-excitation coupled array. The correspondence between the behavior of entropy and phase diagram in the lowest-energy state is established. We find that the entanglement of photons in the photonic superfluid state has an approximately quadratic-like dependence on the total excitation number within the large-detuning limits, and the genuine multipartite entanglement in the polaritonic superfluid state for the large-hopping situation here is more obvious than that in the system with only two excitations. The Mandel Q-parameter is always negative in the lowest-energy state, which is independent of the total excitation number and approaches to -0.5 within the large-detuning limits. It is interesting to find that the insulator states can also be identified as uncorrelated states but the superfluid states are always anticorrelated states. We also analytically demonstrate that the second-order crosscorrelation function is inversely proportional to the total excitation number in both large-detuning limits. 
The results we have presented are not limited to the cavity QED system, and are general and applicable to all the system$\mathrm{s}$, such as ion traps and circuit QED systems. The physical importance here is to gain more insights in the nature of quantum phase transition for polaritonic excitations.
[1] Lewenstein, M., Sanpera, A., Ahufinger, V., Damski, B., Sen(De), A. and Sen U. Ultracold atomic gases in optical lattices: mimicking condensed matter physics and beyond, Adv. Phys. 56, 243 (2007).

[2] Bloch, I., Dalibard, J. and Zwerger, W. Many-body physics with ultracold gases, Rev. Mod. Phys. 80, 885 (2008).

[3] Fazio, R. and van der Zant, H. Quantum phase transitions and vortex dynamics in superconducting networks, Phys. Rep. 355, 235 (2001)

[4] Greentree, A. D., Tahan, C., Cole, J. H. and Hollenberg, L. C. L. Quantum phase transitions of light, Nature Physics 2, 856 (2006).

[5] Hartmann, M. J., Brandão, F. G. S. L. and Plenio, M. B. Strongly interacting polaritons in coupled arrays of cavities, Nature Physics 2, 849 (2006).

[6] Angelakis, D. G., Santos, M. F. and Bose, S. Photon-blockadeinduced Mott transitions and XY spin models in coupled cavity arrays, Phys. Rev. A 76, 031805 (2007).

[7] van der Zant, H. S. J., Fritschy, F. C., Elion, W. J., Geerligs, L. J. and Mooij, J. E. Field-induced superconductor-to-insulator transitions in Josephson-junction arrays, Phys. Rev. Lett. 69, 2971 (1992).

[8] Jaksch, D., Bruder, C., Cirac, J. I., Gardiner, C. W. and Zoller, P. Cold bosonic atoms in optical lattices, Phys. Rev. Lett. 81, 3108 (1997).

[9] Imamoglu, A., Schmidt, H., Woods, G. and Deutsch, M. Strongly interacting photons in a nonlinear cavity, Phys. Rev. Lett. 79, 1467 (1997).

[10] Birnbaum, K. M., Boca, A., Miller, R., Boozer, A. D., Northup, T. E. and Kimble, H. J. Photon blockade in an optical cavity with one trapped atom, Nature (London) 436, 87 (2005).

[11] Greiner, M., Mandel, O., Esslinger, T., Hansch, T. W. and Bloch, I. Quantum phase transition from a superfluid to a Mott insulator in a gas of ultracold atoms, Nature (London) 415, 39 (2002).

[12] Raimond, J. M., Brune, M. and Haroche, S. Manipulating quantum entanglement with atoms and photons in a cavity, Rev. Mod. Phys. 73, 565 (2001).

[13] Hennessy, K., Badolato, A., Winger, M., Gerace, D., Atatürel, M., Gulde, S., Fält, S., Hu, E. L. and Imamoğlu, A. Quantum nature of a strongly coupled single quantum dot-cavity system, Nature 445, 896 (2007).

[14] Ashhab, S. Superradiance transition in a system with a single qubit and a single oscillator, Phys. Rev. A 87, 013826 (2013).

[15] Makin, M. I., Cole, J. H., Tahan, C., Hollenberg, L. C. L. and Greentree, A. D. Quantum phase transitions in photonic cavities with two-level systems, Phys. Rev. A 77, 053819 (2008).

[16] Rossini, D. and Fazio, R. Mott-Insulating and glassy phases of polaritons in 1D arrays of coupled cavities, Phys. Rev. Lett. 99, 186401 (2007)

[17] Knap, M., Arrigoni, E. and von der Linden, W. Quantum phase transition and excitations of the Tavis-Cummings lattice model, Phys. Rev. B 82, 045126 (2010).

[18] Huo, M. X., Li, Y., Song, Z. and Sun, C. P. Atomic entanglement versus visibility of photon interference for quantum criticality of a hybrid system, Phys. Rev. A 77, 022103 (2008).
[19] Zheng, S. B., Dicke-like quantum phase transition and vacuum entanglement with two coupled atomic ensembles, Phys. Rev. A 84, 033817 (2011).

[20] Irish, E. K., Ogden, C. D. and Kim, M. S. Polaritonic characteristics of insulator and superfluid states in a coupled-cavity array, Phys. Rev. A 77, 033801 (2008).

[21] Irish, E. K. Ground-state entanglement in a coupled-cavity model, Phys. Rev. A 80, 043825 (2009).

[22] Lühmann, D. S., Bongs, K., Sengstock, K. and Pfannkuche, D. Localization and delocalization of ultracold bosonic atoms in finite optical lattices, Phys. Rev. A 77, 023620 (2008).

[23] Tan, L., Zhang, Y. Q. and Liu, W. M. Quantum phase transitions for two coupled cavities with dipole-interaction atoms, Phys. Rev. A 84, 063816 (2011).

[24] Ivanov, P. A., Ivanov, S. S., Vitanov, N. V., Mering, A., Fleischhauer, M. and Singer, K. Simulation of a quantum phase transition of polaritons with trapped ions, Phys. Rev. A 80, 060301 (2009).

[25] Hwang, M. J. and Plenio, M. B. Quantum phase transition in the finite Jaynes-Cummings lattice systems, Phys. Rev. Lett. 117, 123602 (2016).

[26] Hwang, M. J., Puebla, R. and Plenio, M. B. Quantum phase transition and universal dynamics in the Rabi model, Phys. Rev. Lett. 115, 180404 (2015).

[27] Vidal, J. and Dusuel, S. Finite-size scaling exponents in the Dicke model, Europhys. Lett. 74, 817 (2006).

[28] Braak, D. Integrability of the Rabi model, Phys. Rev. Lett. 107, 100401 (2011)

[29] Larson, J. Dynamics of the Jaynes-Cummings and Rabi models: old wine in new bottles, Phys. Scr. 76, 146 (2007).

[30] Emary, C. and Brandes, T. Quantum chaos triggered by precursors of a quantum phase transition: the Dicke model, Phys. Rev. Lett. 90, 044101 (2003).

[31] Bakemeier, L., Alvermann, A. and Fehske, H. Quantum phase transition in the Dicke model with critical and noncritical entanglement, Phys. Rev. A 85, 043821 (2012).

[32] Fink, J. M., Dombi, A., Vukics, A., Wallraff, A. and Domokos, P. Observation of the photon-blockade breakdown phase transition, arXiv: 1607.04892, (2016).

[33] Zhu, G., Schmidt, S. and Koch, J. Dispersive regime of the Jaynes-Cummings and Rabi lattice, New J. Phys. 15, 115002 (2013).

[34] Baksic, A. and Ciuti, C. Controlling discrete and continuous symmetries in "superradian" phase transitions with circuit QED systems, Phys. Rev. Lett. 112, 173601 (2014).

[35] Cirac, J. I., Parkins, A. S., Blatt, R. and Zoller, P. "Dark" squeezed states of the motion of a trapped ion, Phys. Rev. Lett. 70, 556 (1993)

[36] Chen, Q. H., Wang, C., He, S., Liu, T. and Wang, K. L. Exact solvability of the quantum Rabi model using Bogoliubov operators, Phys. Rev. A 86, 023822 (2012).

[37] Zhong, H. H., Xie, Q. T., Batchelor, M. T. and Lee, C. H. Analytical eigenstates for the quantum Rabi model, J. Phys. A 46, 415302 (2013).

[38] Du, L. H., Zhou, X. F., Zhou, Z. W., Zhou, X. and Guo, G. C. Generalized Rabi model in quantum-information processing in- 
cluding the $A^{2}$ term, Phys. Rev. A 86, 014303 (2012).

[39] Zheng, H. Dynamics of a two-level system coupled to Ohmic bath: a perturbation approach, Eur. Phys. J. B 38, 559 (2004).

[40] Xie, Q. T., Cui, S., Cao, J. P., Amico, L. and Fan, H. Anisotropic Rabi model, Phys. Rev. X 4, 021046 (2014).

[41] Wang, Z. H., Zheng, Q., Wang, X. G. and Li, Y. The energylevel crossing behavior and quantum Fisher information in a quantum well with spin-orbit coupling, Sci. Rep. 6, 22347 (2016).

[42] Zhang, G. F. and Zhu, H. J. Analytical solution for the anisotropic Rabi model: effects of counter-rotating terms, Sci. Rep. 5, 8756 (2015).

[43] Zhang, Y. Y. Generalized squeezing rotating-wave approximation to the isotropic and anisotropic Rabi model in the ultrastrong-coupling regime, Phys. Rev. A 94, 063824 (2016).

[44] De Grandi, C., Gritsev, V. and Polkovnikov, A. Quench dynamics near a quantum critical point, Phys. Rev. B 81, 012303 (2010).

[45] Liu, Y. X., Yang, C. X., Sun, H. C. and Wang, X. B. Coexistence of single- and multi-photon processes due to longitudinal couplings between superconducting flux qubits and external fields. New J. Phys. 16, 015031 (2014).

[46] Yang, C. P., Chu, S. I. and Han, S. Possible realization of entanglement, logical gates, and quantum-information transfer with superconducting-quantum-interference-device qubits in cavity QED. Phys. Rev. A 67, 042311 (2003).

[47] Chen, G., Xue, Z. Y., Wei, L. F. and Liang, J. Q. Interactioninduced topological quantum interference in an extended Dicke model, Europhys. Lett. 86, 44002 (2009).

[48] Zhu, S. L., Wang, Z. and Zanardi, P. Geometric quantum computation and multiqubit entanglement with superconducting qubits inside a cavity, Phys. Rev. Lett. 94, 100502 (2005).

[49] Shapourian, H. and Sadri, D. Fock-space localization of polaritons in the Jaynes-Cummings dimer model, Phys. Rev. A 93, 013845 (2016).

[50] Schmidt, S., Gerace, D., Houck, A. A., Blatter, G., and Türeci, H. E. Nonequilibrium delocalization-localization transition of photons in circuit quantum electrodynamics, Phys. Rev. B 82, 100507(R) (2010).

[51] Raftery, J., Sadri, D., Schmidt, S., Türeci, H. E., and Houck, A. A. Observation of a dissipation-induced classical to quantum transition, Phys. Rev. X 4, 031043 (2014).

[52] Koch, J. and Le Hur, K. SuperfluidšCMott-insulator transition of light in the Jaynes-Cummings lattice, Phys. Rev. A 80,
023811 (2009).

[53] Schmidt, S. and Blatter, G. Excitations of strongly correlated lattice polaritons, Phys. Rev. Lett. 104, 216402 (2010).

[54] Schiró, M., Bordyuh, M., Öztop, B., and Türeci, H. E. Phase transition of light in cavity QED lattices, Phys. Rev. Lett. 109, 053601 (2012).

[55] Schiró, M., Joshi, C., Bordyuh, M., Fazio, R., Keeling, J., and Türeci, H. E. Exotic attractors of the nonequilibrium RabiHubbard model, Phys. Rev. Lett. 116, 143603 (2016).

[56] Hafezi, M., Adhikari, P., and Taylor, J. M. Chemical potential for light by parametric coupling, Phys. Rev. B 92, 174305 (2015).

[57] Toyoda, K., Matsuno, Y., Noguchi, A., Haze, S. and Urabe, S. Experimental realization of a quantum phase transition of polaritonic excitations, Phys. Rev. Lett. 111, 160501 (2013).

\section{ACKNOWLEDGEMENTS}

We would like to thank Dr. Kenji Toyoda for useful discussions. EKI acknowledges funding from the Leverhulme Trust. This work is supported by the National Natural Science Foundation of China (Grant Nos. 11674060 and 11705030), the Natural Science Foundation of Fujian Province (Grant No. 2017J05005), the fund from Fuzhou University (Grant Nos. XRC-1566).

\section{AUTHOR CONTRIBUTIONS STATEMENT}

S.B.Z. contributed the idea. L.T.S performed the calculations and prepared the figures. L.T.S. wrote the main manuscript. E.K.I. provided the method. R.X.C., H.Z.W. and Z.B.Y. checked the calculations and made an improvement of the manuscript. All authors contributed to discussion and reviewed the manuscript.

\section{ADDITIONAL INFORMATION}

Competing financial interests: The authors declare no competing financial interests. 